\title{
Nachruf auf Judith le Soldat
}

\author{
Thomas von Salis (Zürich)
}

Judith le Soldat ist am 22. Mai 2008 nach einem schweren Leiden, von dem sie sich nichts hat anmerken lassen, gestorben.

«Nichts Schlimmeres kann man freilich einem toten Dichter antun», schreibt sie 1992 in der NZZ in Bezug auf einen ungarischen Dichter, «als sein Werk von der Warte seiner Vita aus zu betrachten. Menschliches Leid ist kein literarisches Kriterium. Und das erschrockene Mitgefühl, das rasch mit idealisierendem Lob Distanz schaffen will, ist kein guter Standort, um das Geschriebene, im Überlieferten des Menschen, die Zeit, und was sie beide bewegte, zu verstehen.»

Lassen wir also das Biografische der Verstorbenen und schauen auf ihr berufliches und wissenschaftliches Werk.

Dem PSZ seit dessen Anfängen zugehörig, schrieb sie im Journal Nr. 13 eine Entgegnung zu einem in der vorhergehenden Nummer erschienenen kritischen Aufsatz über Morgenthaler: «... Auch ich gehöre zu jenem unbelehrbaren Haufen von Mitstreitern und Schülern Morgenthalers ... wir haben kein spontan funktionierendes «soziokulturelles` Gewissen, halten weder für moralische noch für die psychoanalytische Kulanz, sind, wenn man's recht bedenkt, von Berufes wegen unduldsam, eine Beleidigung für jeden aufrechten Anständigen.» Morgenthaler, schreibt sie, «rekonstruierte ... die ganz besonderen Triebschicksale, die zur normalen männlichen Homosexualität führen. ... Es ist sein grosses Verdienst, uns gelehrt zu haben, dass nicht diese unsere therapeutische Aufmerksamkeit verdienen ... sondern die vielfältigen neurotischen und gesellschaftlichen Konflikte, die diese stören und einebnen wollen.» ${ }^{1}$

In ihrem Psyche-Artikel vom August 2000, «Der Strich des Apelles», kommt sie «am Ende einer einprägsamen Rekonstruktion der Arbeit des Unbewussten bei ihrem Patienten wie bei ihr selbst einer zentralen homosexuellen Phantasie und deren Folgen auf die Spur ${ }^{2}$.

In der spannenden Entrollung der Analyse kommt sie zum Schluss, dass der Maler Apelles in einem sublimierten Streich den "Kreislauf» beendet hatte, jenen «endlosen Kreislauf von Triumph und Verzweiflung, von Selbstvorwürfen und Provokationen, von Leugnung, Wiedergutmachung und lähmender Resignation, aus dem (der Analysand) allein nicht mehr herausfand». - Apelles ist eine antike Figur, ein berühmter Maler, der mit seinem besonders feinen Strich auf eine begon- 
nene Arbeit seines Maler-Kollegen Protogenes einen so gut wie unsichtbaren Strich anbringt, um dem abwesenden Kollegen ein Zeichen zu hinterlassen, dass er da gewesen sei. Protogenes applizierte sodann einen noch feineren Strich daneben, worauf Apelles wieder vergeblich einen Besuch machen will und einen dritten, noch feineren Strich in den Zwischenraum setzt.

J. Le Soldat verhilft diese Legende zur Deutung der besonderen Verlustproblematik ihres homosexuellen Patienten.

Es gelingt ihr, die eigene, ihr vorerst nur vage bewusste und völlig unverständliche Wut auf den Analysanden als identifikatorische Übernahme des verdrängten Affekts des Patienten zu erfassen. Dessen Zustand vermittelt sie mit folgendem Bild: «Der Affekt ist ... aus allen ... Verankerungen herausgerissen, die Inhalte sind weitgehend ihres affektiven Sinnes entkleidet, wie Tote, denen man die Ausweispapiere nimmt, um sie unkenntlich zu machen.»

Ihre Technik, wie sie sie um 1990 beschreibt, entspricht m.E. in einem gewissen Sinne dem, was heute als beste Ausformung der nordamerikanischen interaktionistischen («Enactment») Analysentechnik gilt: vgl. dazu Lawrence Friedman: «Ritorno agli articoli di Freud sulla tecnica.» ${ }^{3}$ Sie hält sich an die Regeln der Abstinenz und der Diskretion und lässt sich gleichzeitig in das emotionale Geschehen in der Beziehung mit den Analysanden ein, sodass sie in die Lage kommt, die Gegenübertragung zur Analyse des Übertragungsgeschehens zu verwenden. Abstinenz kann hier gemäss der (ein wenig erweiterten) Definition von Anna Freud verstanden werden: Gleiche Aufmerksamkeit wird den 4 Instanzen, Umwelt, Ich, Es und Überich, zuteil, sowohl beim Analysanden als auch beim Analytiker.

Zum Verhältnis zwischen Individuum und Gesellschaft zitiert sie Parin und Morgenthaler: «Die Dogon sind ein Volk, dem eineVerarbeitung der Aggression ohne Verinnerlichung gelingt, ohne dass sozial unerwünschte Wirkungen der Aggression auftreten. (...) Bei den Agni kommt es ebenfalls nicht zu einer Introjektion der Autorität des Vaters.» (...) Anstatt einer inneren, moralischen Instanz bilde sich ein sog. «Clan-Gewissen» heraus. Ich denke aber (fährt Le Soldat fort), dass Parin und Morgenthaler bereits die Folgen einer Reprojektion, einer sekundären Abwehr der Überichbildung beobachtet haben. Die aktive, gegen den Vater (bzw. das erste männliche Liebesobjekt) gewendete Kastrationslust erachte ich als eine unausweichliche Konsequenz der psycho-sexuellen Triebentwicklung. Sie ist die genitale Modalität der Aggression, insofern obligat. Ihre primären Ziele sind daher auch (wie der Aggressionstrieb an sich) kultur-unabhängig. Von gesellschaftlichen Bedingungen unabhängig ist ebenso die konfliktuöse Verstrickung der beiden 
Triebarten, von Libido und Aggression während der ödipalen Krise. Es ist dies ein triebdynamischer Prozess, der notwendigerweise und ohne jeglichen äusseren Einfluss mit einer Abspaltung und einer Funktionsänderung eines Teils des Aggressionspotentials (des Überichs) endet. Phänomenologisch sein Resultat beschreibend, nennen wir den Vorgang «Verinnerlichung». Der ödipale Sadismus richtet sich auch aus triebimmanenten und nicht aus sozialen Gründen gegen das erste männliche Liebesobjekt (und keineswegs gegen einen Rivalen), das mit genitaler Erregung begehrt wurde. Jedes männliche Objekt, notfalls auch ein weibliches, dem man einen Phallus unterstellt, ist gut genug, die unvermeidlichen Projektionen zu tragen. Die Frage des Inzests und der Rivalität sind dagegen zweitrangig, kommen später, sind bereits als Lösungen des aufgrund des Triebanspruchs entstandenen Konflikts kulturspezifisch und variabel (4).

Es geht in der von Le Soldat in ihrem Text «Kissing and Killing in Kyoto» beschriebenen Symptomatik um «Lärmende, agitierte Versuche», dem entstandenen Dilemma zu entkommen. Versuche, die innere Zwangslage aussen, das innere, psychische Elend in der realen Aussenwelt zu heilen. Erbitterte Streifzüge, der äusseren Realität doch noch abzuringen, was das Innere nicht mehr hergibt. Diese in jeder Hinsicht komplexe, vielfach gebrochene Form der Äusserung des Sadismus tritt nun, so meine ich, nicht selten an die Stelle eines simplen aggressiven Triebablaufes, ersetzt manchmal auch die (vom postödipalen Standpunkt aus gesehen) regressiven Formen von Raubgier, Mordlust und Rachsucht, und macht uns so viel Mühe bei der Auffassung wie auch bei ihrer Beherrschung. Denn nun hasst man, bedrängt, foltert und vergewaltigt andere, deren Schmerzen man ja nicht empfindet, um - mit der genannten Wendung vom passiven Wunsch zur Aktivität - an der phantasierten Wunschbefriedigung zumindest identifikatorisch noch partizipieren zu können. Man randaliert und mordet, weil man besinnungslos und blindlings demonstriert, was man für sich wünscht, wenn man es bloss wagen könnte: Dass sich jemand auf einen stürzen würde, so entflammt von Liebe und Begierde, so hingerissen, so leidenschaftlich und gewalttätig, wie Goliath auf David, wie Penthesilea auf Achill, ganz gleichgültig gegenüber den Grenzen des Körpers ... Sobald der innere Konflikt schärfer wird und die Neigung zum Agieren grösser, stürzt man sich nur noch aus Rache und Frustration auf jeden, der es hätte tun können, und es doch nicht tut. Man rächt sich aus Neid an denen, die vermeintlich die passiven Wonnen, von denen man sich ausgeschlossen wähnt, geniessen: an den Frauen, den Schwulen. Oder man versucht die Identifikation mit dem Aggressor, bringt die eigenen Wünsche zu Fall, die dann prompt als Hoffnungen auf Rettung und Erlösung durch einen ‘grossen Mann` wiederkehren.» ${ }^{5}$ 
Und an einem anderen Ort:

«... die Geschichte der Masochismus-Theorie zeigt, wie sehr das seelische Geschehen ausschliesslich vom Luststreben bestimmt wird. Allerdings wird man sich mit der Aussicht befreunden müssen, dass auch Aggressions- und Destruktionswünsche in jedem Fall Lust suchen und Lust bringen. Ich habe zu zeigen versucht, dass die Entwicklung von Freuds Ideen zum Problem des Masochismus als die Geschichte des Ringens um das Bewusstsein der Ubiquität der triebhaften Aggression gelesen werden kann. Der Weg von der ursprünglichen Idee der Abwehr eines sadistischen Impulses zu genuinen masochistischen Strebungen in der Todestriebtheorie will uns als das Sträuben gegen die Einsicht erscheinen, dass auch die Macht des Todestriebes eben jene einer simplen lustvollen Triebbefriedigung ist.» ${ }^{6}$

Auf der Homepage www.lesoldat.chlassen sich Einblicke in das Werk Judith Le Soldats gewinnen.

Leider war es ihr nicht mehr möglich, die von ihr geplanten und schon weit fortgeschrittenen Publikationen - eine Weiterentwicklung ihrer Theorie, die im Buch «Eine Theorie menschlichen Unglücks» angelegt ist, sowie ihre weiter ausgearbeitete Theorie der Homosexualität - fertig zu stellen. Eine postume Herausgabe ist vorgesehen.

Einige wenige Exemplare der beiden vergriffenen Bücher wurden dem PSZ geschenkt. Sie können dort erworben werden.

Wir haben an Judith Le Soldat eine überaus kreative, hochgeschätzte Kollegin verloren.

Auf ihrem Schreibtisch fand sich auf einem Post-it-Zettelchen die Notiz: «Non omnis moriar ...»

\section{Literatur}

Le Soldat, J. (1985): Journal des PSZ (Psychoanalytisches Seminar Zürich) Nr. 13, November 1985, 30-32.

Le Soldat, J. (2000): Psyche 54, 742, aus der Übersicht. Es handelt sich bei dieser Publikation um die Wiedergabe eines Vortrags von 1988.

Friedman, L. (2008): Ritorno agli articoli di Freud sulla tecnica. In: Psicoterapia e Scienze umane 2, 151-162.

Le Soldat, J. (1994): Eine Theorie menschlichen Unglücks, Frankfurt /M. (Sozialwissenschaft Fischer), Anmerkungsteil, 351-352.

Le Soldat, J. (2001): Kissing and Killing in Kyoto. In: Klöpper M., Lindner R. (Hrsg.), Destruktivität. Wurzeln und Gesichter. Göttingen: Vandenhoek \& Ruprecht, 133.

Le Soldat, J. (1986): Psyche 40, 638. 